\title{
SppC Based Energy Frontier Lepton-Proton Colliders: Luminosity and Physics
}

\author{
Ali Can Canbay, ${ }^{1,2}$ Umit Kaya, ${ }^{1,2}$ Bora Ketenoglu, ${ }^{3}$ \\ Bilgehan Baris Oner, ${ }^{1}$ and Saleh Sultansoy ${ }^{1,4}$ \\ ${ }^{1}$ TOBB University of Economics and Technology, Ankara, Turkey \\ ${ }^{2}$ Department of Physics, Ankara University, Ankara, Turkey \\ ${ }^{3}$ Department of Engineering Physics, Ankara University, Ankara, Turkey \\ ${ }^{4}$ ANAS Institute of Physics, Baku, Azerbaijan \\ Correspondence should be addressed to Umit Kaya; umit.kaya@cern.ch
}

Received 14 April 2017; Accepted 15 June 2017; Published 1 August 2017

Academic Editor: Juan José Sanz-Cillero

Copyright (C) 2017 Ali Can Canbay et al. This is an open access article distributed under the Creative Commons Attribution License, which permits unrestricted use, distribution, and reproduction in any medium, provided the original work is properly cited. The publication of this article was funded by SCOAP ${ }^{3}$.

\begin{abstract}
Main parameters of Super proton-proton Collider (SppC) based lepton-proton colliders are estimated. For electron beam parameters, highest energy International Linear Collider (ILC) and Plasma Wake Field Accelerator-Linear Collider (PWFA-LC) options are taken into account. For muon beams, $1.5 \mathrm{TeV}$ and $3 \mathrm{TeV}$ center of mass energy muon collider parameters are used. In addition, ultimate $\mu p$ collider which assumes construction of additional $50 \mathrm{TeV}$ muon ring in the SppC tunnel is considered. It is shown that luminosity values exceeding $10^{32} \mathrm{~cm}^{-2} \mathrm{~s}^{-1}$ can be achieved with moderate upgrade of the SppC proton beam parameters. Physics search potential of proposed lepton-proton colliders is illustrated by considering small Björken $x$ region as an example of SM physics and resonant production of color octet leptons as an example of BSM physics.
\end{abstract}

\section{Introduction}

It is known that lepton-hadron scattering had played crucial role in our understanding of deep inside of matter. For example, electron scattering on atomic nuclei reveals structure of nucleons in Hofstadter experiment [1]. Moreover, quark parton model was originated from lepton-hadron collisions at SLAC [2]. Extending the kinematic region by two orders of magnitude both in high $Q^{2}$ and small $x$, HERA (the first and still unique lepton-hadron collider) with $\sqrt{s}=0.32 \mathrm{TeV}$ has shown its superiority compared to the fixed target experiments and provided parton distribution functions (PDF) for LHC and Tevatron experiments (for review of HERA results see $[3,4])$. Unfortunately, the region of sufficiently small $x\left(<10^{-5}\right)$ and high $Q^{2}\left(\geq 10 \mathrm{GeV}^{2}\right)$ simultaneously, where saturation of parton densities should manifest itself, has not been reached yet. Hopefully, $\mathrm{LHeC}[5]$ with $\sqrt{s}=1.3 \mathrm{TeV}$ will give opportunity to touch this region.

Construction of linear $e^{+} e^{-}$colliders (or dedicated linac) and muon colliders (or dedicated muon ring) tangential to the future circular pp colliders, FCC or SppC, as shown in Figure 1, will give opportunity to use highest energy proton beams in order to obtain highest center of mass energy in lepton-hadron and photon-hadron collisions (for earlier studies on linac-ring type $e p, \gamma p, e A$, and $\gamma A$ colliders, see reviews $[6,7]$ and papers $[8-14])$.

FCC is the future $100 \mathrm{TeV}$ center of mass energy pp collider studied at CERN and supported by European Union within the Horizon 2020 Framework Programme for Research and Innovation [15]. SppC is the Chinese analog of the FCC. Main parameters of the SppC proton beam $[16,17]$ are presented in Table 1 . The FCC based $e p$ and $\mu p$ colliders have been considered recently (see [18] and references therein).

In this paper we consider SppC based $e p$ and $\mu p$ colliders. In Section 2, main parameters of proposed colliders, namely, center of mass energy and luminosity, are estimated taking into account beam-beam tune shift and disruption effects. Physics search potential of the SppC based $l p$ colliders have been evaluated in Section 3, where small Björken $x$ region 


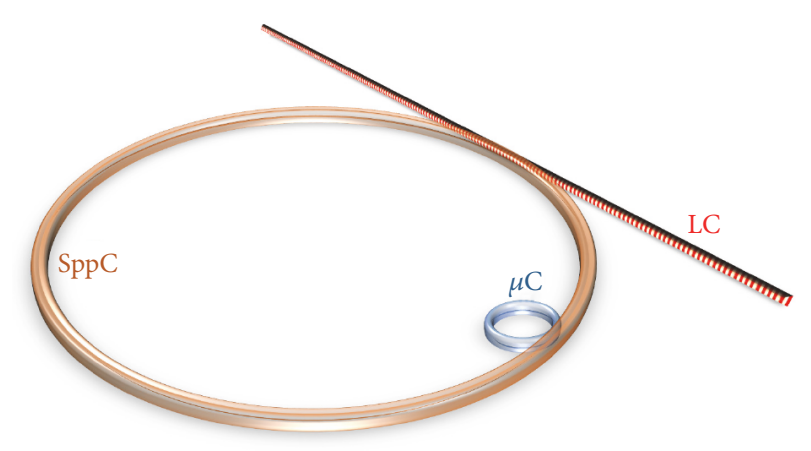

FIgURE 1: Possible configuration for SppC, linear collider (LC), and muon collider $(\mu \mathrm{C})$.

TABLE 1: Main parameters of proton beams in SppC.

\begin{tabular}{lcc}
\hline Beam energy $(\mathrm{TeV})$ & 35.6 & 68.0 \\
Circumference $(\mathrm{km})$ & 54.7 & 100.0 \\
Peak luminosity $\left(10^{34} \mathrm{~cm}^{-2} \mathrm{~s}^{-1}\right)$ & 11 & 102 \\
Particle per bunch $\left(10^{10}\right)$ & 20 & 20 \\
Norm. transverse emittance $(\mu \mathrm{m})$ & 4.10 & 3.05 \\
$\beta^{*}$ amplitude function at IP $(\mathrm{m})$ & 0.75 & 0.24 \\
IP beam size $(\mu \mathrm{m})$ & 9.0 & 3.04 \\
Bunches per beam & 5835 & 10667 \\
Bunch spacing $(\mathrm{ns})$ & 25 & 25 \\
Bunch length $(\mathrm{mm})$ & 75.5 & 15.8 \\
Beam-beam parameter, $\xi_{p p}$ & 0.006 & 0.008 \\
\hline
\end{tabular}

is considered as an example of the SM physics and resonant production of color octet leptons is considered as an example of the BSM physics. Our conclusions and recommendations are presented in Section 4.

\section{Main Parameters of the SppC Based ep and $\mu p$ Colliders}

General expression for luminosity of SppC based $l p$ colliders is given by ( $l$ denotes electron or muon)

$$
L_{l p}=\frac{N_{l} N_{p}}{4 \pi \max \left[\sigma_{x_{p}}, \sigma_{x_{l}}\right] \max \left[\sigma_{y_{p}}, \sigma_{y_{l}}\right]} \min \left[f_{c_{p}}, f_{c_{l}}\right],
$$

where $N_{l}$ and $N_{p}$ are numbers of leptons and protons per bunch, respectively; $\sigma_{x_{p}}\left(\sigma_{x_{l}}\right)$ and $\sigma_{y_{p}}\left(\sigma_{y_{l}}\right)$ are the horizontal and vertical proton (lepton) beam sizes at interaction point (IP); $f_{c_{l}}$ and $f_{c_{p}}$ are LC/ $\mu \mathrm{C}$ and SppC bunch frequencies. $f_{c}$ is expressed by $f_{c}=N_{b} f_{\text {rep}}$, where $N_{b}$ denotes number of bunches and $f_{\text {rep }}$ means revolution frequency for SppC/ $\mu \mathrm{C}$ and pulse frequency for LC. In order to determine collision frequency of $l p$ collider, minimum value should be chosen among lepton and hadron bunch frequencies. Some of these parameters can be rearranged in order to maximize $L_{l p}$ but one should note that there are main limitations due to beambeam effects that should be kept in mind. While beam-beam tune shift affects proton and muon beams, disruption has influence on electron beams.
Disruption parameter for electron beam is given by

$$
\begin{aligned}
& D_{x_{e}}=\frac{2 N_{p} r_{e} \sigma_{z_{p}}}{\gamma_{e} \sigma_{x_{p}}\left(\sigma_{x_{p}}+\sigma_{y_{p}}\right)}, \\
& D_{y_{e}}=\frac{2 N_{p} r_{e} \sigma_{z_{p}}}{\gamma_{e} \sigma_{y_{p}}\left(\sigma_{y_{p}}+\sigma_{x_{p}}\right)},
\end{aligned}
$$

where $r_{e}=2.82 \times 10^{-15} \mathrm{~m}$ is classical radius for electron, $\gamma_{e}$ is the Lorentz factor of electron beam, and $\sigma_{x_{p}}$ and $\sigma_{y_{p}}$ are horizontal and vertical proton beam sizes at IP, respectively. $\sigma_{z_{p}}$ is bunch length of proton beam. Beam-beam parameter for proton beam is given by

$$
\begin{aligned}
& \xi_{x_{p}}=\frac{N_{l} r_{p} \beta_{p}^{*}}{2 \pi \gamma_{p} \sigma_{x_{l}}\left(\sigma_{x_{l}}+\sigma_{y_{l}}\right)}, \\
& \xi_{y_{p}}=\frac{N_{l} r_{p} \beta_{p}^{*}}{2 \pi \gamma_{p} \sigma_{y_{l}}\left(\sigma_{y_{l}}+\sigma_{x_{l}}\right)},
\end{aligned}
$$

where $r_{p}$ is classical radius for proton, $r_{p}=1.54 \times 10^{-18} \mathrm{~m}$, $\beta_{p}^{*}$ is beta function of proton beam at IP, and $\gamma_{p}$ is the Lorentz factor of proton beam. $\sigma_{x_{l}}$ and $\sigma_{y_{l}}$ are horizontal and vertical sizes of lepton beam at IP, respectively.

Beam-beam parameter for muon beam is given by

$$
\begin{aligned}
& \xi_{x_{\mu}}=\frac{N_{p} r_{\mu} \beta_{\mu}^{*}}{2 \pi \gamma_{\mu} \sigma_{x_{p}}\left(\sigma_{x_{p}}+\sigma_{y_{p}}\right)}, \\
& \xi_{y_{\mu}}=\frac{N_{p} r_{\mu} \beta_{\mu}^{*}}{2 \pi \gamma_{\mu} \sigma_{y_{p}}\left(\sigma_{y_{p}}+\sigma_{x_{p}}\right)},
\end{aligned}
$$

where $r_{\mu}=1.37 \times 10^{-17} \mathrm{~m}$ is classical muon radius, $\beta_{\mu}^{*}$ is beta function of muon beam at IP, and $\gamma_{\mu}$ is the Lorentz factor of muon beam. $\sigma_{x_{p}}$ and $\sigma_{y_{p}}$ are horizontal and vertical sizes of proton beam at IP, respectively.

2.1. ep Option. Preliminary study of CepC-SppC based $e-p$ collider with $\sqrt{s}=4.1 \mathrm{TeV}$ and $L_{e p}=10^{33} \mathrm{~cm}^{-2} \mathrm{~s}^{-1}$ has been performed in [19]. In this subsection, we consider ILC (International Linear Collider) [20] and PWFA-LC (Plasma Wake Field Accelerator-Linear Collider) [21] as a source of electron/positron beam for SppC based energy frontier ep colliders. Main parameters of ILC and PWFA-LC electron beams are given Table 2 .

It is seen that bunch spacings of ILC and PWFA-LC are much greater than SppC bunch spacing. On the other hand, transverse size of proton beam is much greater than transverse sizes of electron beam. Therefore, (1) for luminosity turns into

$$
L_{e p}=\frac{N_{e} N_{p}}{4 \pi \sigma_{p}^{2}} f_{c_{e}}
$$


TABLE 2: Main parameters of the ILC (second column) and PWFALC (third column) electron beams.

\begin{tabular}{lcc}
\hline Beam energy $(\mathrm{GeV})$ & 500 & 5000 \\
Peak luminosity $\left(10^{34} \mathrm{~cm}^{-2} \mathrm{~s}^{-1}\right)$ & 4.90 & 6.27 \\
Particle per bunch $\left(10^{10}\right)$ & 1.74 & 1.00 \\
Norm. horiz. emittance $(\mu \mathrm{m})$ & 10.0 & 10.0 \\
Norm. vert. emittance $(\mathrm{nm})$ & 30.0 & 35.0 \\
Horiz. $\beta^{*}$ amplitude function at IP $(\mathrm{mm})$ & 11.0 & 11.0 \\
Vert. $\beta^{*}$ amplitude function at IP $(\mathrm{mm})$ & 0.23 & 0.099 \\
Horiz. IP beam size $(\mathrm{nm})$ & 335 & 106 \\
Vert. IP beam size $(\mathrm{nm})$ & 2.70 & 59.8 \\
Bunches per beam & 2450 & 1 \\
Repetition rate $(\mathrm{Hz})$ & 4.00 & 5000 \\
Beam power at IP $(\mathrm{MW})$ & 27.2 & 40 \\
Bunch spacing $(\mathrm{ns})$ & 366 & $20 \times 10^{4}$ \\
Bunch length $(\mathrm{mm})$ & 0.225 & 0.02 \\
\hline
\end{tabular}

TABLE 3: Main parameters of LC $\otimes$ SppC based ep colliders.

\begin{tabular}{lccccc}
\hline$E_{e}, \mathrm{TeV}$ & $E_{p}, \mathrm{TeV}$ & $\sqrt{s}, \mathrm{TeV}$ & $L_{e p}, \mathrm{~cm}^{-2} \mathrm{~s}^{-1}$ & $D_{e}$ & $\xi_{p}, 10^{-3}$ \\
\hline 0.5 & 35.6 & 8.44 & $3.35(6.64) \times 10^{30}$ & 0.537 & 0.5 \\
0.5 & 68 & 11.66 & $2.69(5.33) \times 10^{31}$ & 0.902 & 0.7 \\
5 & 35.6 & 26.68 & $0.98(1.94) \times 10^{30}$ & 0.054 & 0.3 \\
5 & 68 & 36.88 & $0.78(1.56) \times 10^{31}$ & 0.090 & 0.4 \\
\hline
\end{tabular}

For transversely matched electron and proton beams at IP, equations for electron beam disruption and proton beam tune shift become

$$
\begin{aligned}
& D_{e}=\frac{N_{p} r_{e} \sigma_{z_{p}}}{\gamma_{e} \sigma_{p}^{2}}, \\
& \xi_{p}=\frac{N_{e} r_{p} \beta_{p}^{*}}{4 \pi \gamma_{p} \sigma_{p}^{2}}=\frac{N_{e} r_{p}}{4 \pi \epsilon_{n p}},
\end{aligned}
$$

where $\epsilon_{n p}$ is normalized transverse emittance of proton beam.

Using nominal parameters of ILC, PWFA-LC, and SppC, we obtain values of $L_{e p}, D_{e}$, and $\xi_{p}$ parameters for LC $\otimes$ SppC based ep colliders, which are given in Table 3 . The values for luminosity given in parentheses represent results of beambeam simulations by ALOHEP software [22], which is being developed for linac-ring type ep colliders.

In order to increase luminosity of ep collisions LHeClike upgrade of the SppC proton beam parameters has been used. Namely, $\beta$ function of proton beam at IP is arranged to be $7.5 / 2.4$ times lower $(0.1 \mathrm{~m}$ instead of $0.75 / 0.24 \mathrm{~m})$ which corresponds to LHeC [5] and THERA [23] designs. This leads to increase of luminosity and $D_{e}$ by factor 7.5 and 2.4 for SppC with $35.6 \mathrm{TeV}$ and $68 \mathrm{TeV}$ proton beam, respectively. Results are shown in Table 4.

In principle "dynamic focusing scheme" [24], which was proposed for THERA, could provide additional factor of 34. Therefore, luminosity values exceeding $10^{32} \mathrm{~cm}^{-2} \mathrm{~s}^{-1} \mathrm{can}$ be achieved for all options. Concerning ILC $\otimes S p p C$ based ep colliders, a new scheme for energy recovery proposed for higher-energy LHeC (see Section 7.1.5 in [5]) may give an
TABLE 4: Main parameters of LC $\otimes S p p C$ based ep colliders with upgraded $\beta^{*}$.

\begin{tabular}{lccccc}
\hline$E_{e}, \mathrm{TeV}$ & $E_{p}, \mathrm{TeV}$ & $\sqrt{s}, \mathrm{TeV}$ & $L_{e p}, \mathrm{~cm}^{-2} \mathrm{~s}^{-1}$ & $D_{e}$ & $\xi_{p}, 10^{-3}$ \\
\hline 0.5 & 35.6 & 8.44 & $2.51(4.41) \times 10^{31}$ & 4.03 & 0.5 \\
0.5 & 68 & 11.66 & $6.45(10.8) \times 10^{31}$ & 2.16 & 0.7 \\
5 & 35.6 & 26.68 & $7.37(13.3) \times 10^{30}$ & 0.403 & 0.3 \\
5 & 68 & 36.88 & $1.89(3.75) \times 10^{31}$ & 0.216 & 0.4 \\
\hline
\end{tabular}

TABLE 5: Main parameters of the muon beams.

\begin{tabular}{lcc}
\hline Beam energy $(\mathrm{GeV})$ & 750 & 1500 \\
Circumference $(\mathrm{km})$ & 2.5 & 4.5 \\
Average luminosity $\left(10^{34} \mathrm{~cm}^{-2} \mathrm{~s}^{-1}\right)$ & 1.25 & 4.4 \\
Particle per bunch $\left(10^{12}\right)$ & 2 & 2 \\
Norm. trans. emitt. $(\mathrm{mm}-\mathrm{rad})$ & 0.025 & 0.025 \\
$\beta^{*}$ amplitude function at IP $(\mathrm{cm})$ & $1(0.5-2)$ & $0.5(0.3-3)$ \\
IP beam size $(\mu \mathrm{m})$ & 6 & 3 \\
Bunches per beam & 1 & 1 \\
Repetition rate $(\mathrm{Hz})$ & 15 & 12 \\
Bunch spacing $(\mathrm{ns})$ & 8300 & 15000 \\
Bunch length $(\mathrm{cm})$ & 1 & 0.5 \\
\hline
\end{tabular}

opportunity to increase luminosity by an additional order, resulting in $L_{e p}$ exceeding $10^{33} \mathrm{~cm}^{-2} \mathrm{~s}^{-1}$. Unfortunately, this scheme can not be applied at PWFA-LC $\otimes$ SppC.

2.2. up Option. Muon-proton colliders were proposed almost two decades ago: construction of additional proton ring in $\sqrt{s}$ $=4 \mathrm{TeV}$ muon collider tunnel was suggested in [25], construction of additional $200 \mathrm{GeV}$ energy muon ring in the Tevatron tunnel was considered in [26], and ultimate $\mu p$ collider with $50 \mathrm{TeV}$ proton ring in $\sqrt{s}=100 \mathrm{TeV}$ muon collider tunnel was suggested in [27]. Here, we consider construction of $\mathrm{TeV}$ energy muon colliders $(\mu \mathrm{C})[28]$ tangential to the SppC. Parameters of $\mu \mathrm{C}$ are given in Table 5 .

Keeping in mind that both SppC and $\mu \mathrm{C}$ have round beams, luminosity equation (1) turns to

$$
\begin{aligned}
& L_{p p}=f_{p p} \frac{N_{p}^{2}}{4 \pi \sigma_{p}^{2}}, \\
& L_{\mu \mu}=f_{\mu \mu} \frac{N_{\mu}^{2}}{4 \pi \sigma_{\mu}^{2}},
\end{aligned}
$$

for SppC- $p p$ and $\mu \mathrm{C}$, respectively. Concerning muon-proton collisions one should use larger transverse beam sizes and smaller collision frequency values. Keeping in mind that $f_{\mu \mu}$ is smaller than $f_{p p}$ by more than two orders, the following correlation between $\mu p$ and $\mu \mu$ luminosities takes place:

$$
L_{\mu p}=\left(\frac{N_{p}}{N_{\mu}}\right)\left(\frac{\sigma_{\mu}}{\max \left[\sigma_{p}, \sigma_{\mu}\right]}\right)^{2} L_{\mu \mu} .
$$

Using nominal parameters of $\mu \mu$ colliders given in Table 5, parameters of the SppC based $\mu p$ colliders are calculated according to (8) and presented in Table 6. Concerning 
TABLE 6: Main parameters of SppC based $\mu p$ colliders.

\begin{tabular}{lccccc}
\hline$E_{\mu}, \mathrm{TeV}$ & $E_{p}, \mathrm{TeV}$ & $\sqrt{ } \mathrm{S}, \mathrm{TeV}$ & $L_{\mu p}, \mathrm{~cm}^{-2} \mathrm{~s}^{-1}$ & $\xi_{\mu}$ & $\xi_{p}$ \\
\hline 0.75 & 35.6 & 10.33 & $5.5 \times 10^{32}$ & $8.7 \times 10^{-3}$ & $6.0 \times 10^{-2}$ \\
0.75 & 68 & 14.28 & $12.5 \times 10^{32}$ & $8.7 \times 10^{-3}$ & $8.0 \times 10^{-2}$ \\
1.5 & 35.6 & 14.61 & $4.9 \times 10^{32}$ & $8.7 \times 10^{-3}$ & $6.0 \times 10^{-2}$ \\
1.5 & 68 & 20.2 & $42.8 \times 10^{32}$ & $8.7 \times 10^{-3}$ & $8.0 \times 10^{-2}$ \\
\hline
\end{tabular}

TABLE 7: Main parameters of the ultimate muon beam.

\begin{tabular}{lc}
\hline Beam energy $(\mathrm{TeV})$ & 50 \\
Circumference $(\mathrm{km})$ & 100 \\
Average luminosity $\left(10^{34} \mathrm{~cm}^{-2} \mathrm{~s}^{-1}\right)$ & 100 \\
Particle per bunch $\left(10^{12}\right)$ & 0.80 \\
Norm. trans. emitt. $(\mathrm{mm}-\mathrm{mrad})$ & 8.7 \\
$\beta^{*}$ amplitude function at IP $(\mathrm{mm})$ & 2.5 \\
IP beam size $(\mu \mathrm{m})$ & 0.21 \\
Bunches per beam & 1 \\
Repetition rate $(\mathrm{Hz})$ & 7.9 \\
Bunch spacing $(\mu \mathrm{s})$ & 333 \\
Bunch length $(\mathrm{mm})$ & 2.5 \\
\hline
\end{tabular}

beam tune shifts, for round and matched beams, (3) and (4) turn to

$$
\begin{aligned}
& \xi_{p}=\frac{N_{\mu} r_{p} \beta_{p}^{*}}{4 \pi \gamma_{p} \sigma_{\mu}^{2}}=\frac{N_{\mu} r_{p}}{4 \pi \epsilon_{n p}}, \\
& \xi_{\mu}=\frac{N_{p} r_{\mu} \beta_{\mu}^{*}}{4 \pi \gamma_{\mu} \sigma_{p}^{2}}=\frac{N_{p} r_{\mu}}{4 \pi \epsilon_{n \mu}},
\end{aligned}
$$

respectively.

As one can see from Table 6, where nominal parameters of SppC proton beam are used, $\xi_{p}$ is unacceptably high and should be decreased to 0.02 which seems acceptable for $\mu p$ colliders [26]. According to (9), $\xi_{p}$ can be decreased, for example, by decrement of $N_{\mu}$ which leads to corresponding reduction of luminosity (three times and four times for $\mu p$ $35.6 \mathrm{TeV}$ and $68 \mathrm{TeV}$, resp.). Alternatively, crab crossing [29] can be used for decreasing of $\xi_{p}$ without change of the luminosity.

2.3. Ultimate up Option. This option can be realized if an additional muon ring is constructed in the SppC tunnel. In order to estimate CM energy and luminosity of $\mu p$ collisions we use muon beam parameters from [30], where $100 \mathrm{TeV}$ center of mass energy muon collider with $100 \mathrm{~km}$ ring circumference has been proposed. These parameters are presented in Table 7.

CM energy, luminosity, and tune shifts for ultimate $\mu p$ collider are given in Table 8 . It is seen that the $\xi_{p}$ value is approximately two times higher than the limiting value 0.02 [26]. This problem can be solved by reducing muon bunch population, which leads to decrease of luminosity by factor of 1.75. Alternatively, crab crossing can be used without change of the luminosity.
TABLE 8: Main parameters of the ultimate SppC based $\mu p$ collider.

\begin{tabular}{cccccc}
\hline$E_{\mu}, \mathrm{TeV}$ & $E_{p}, \mathrm{TeV}$ & $\sqrt{ } \mathrm{S}, \mathrm{TeV}$ & $L_{\mu p}, \mathrm{~cm}^{-2} \mathrm{~s}^{-1}$ & $\xi_{\mu}$ & $\xi_{p}$ \\
\hline 50 & 68 & 116.6 & $1.2 \times 10^{33}$ & $2.6 \times 10^{-2}$ & $3.5 \times 10^{-2}$ \\
\hline
\end{tabular}

TABLE 9: Attainable Björken $x$ values at $Q^{2}=10 \mathrm{GeV}^{2}$.

\begin{tabular}{lcccc}
\hline$E_{l}(\mathrm{TeV})$ & 0.5 & 5 & 1.5 & 50 \\
$x$ & $7 \times 10^{-8}$ & $7 \times 10^{-9}$ & $2 \times 10^{-8}$ & $7 \times 10^{-10}$ \\
\hline
\end{tabular}

\section{Physics}

In order to evaluate physics search potential of the SppC based $l p$ colliders we consider two phenomena; namely, small Björken $x$ region is considered as an example of the SM physics and resonant production of color octet electron and muon is considered as an example of the BSM physics.

3.1. Small Björken $x$. As mentioned above, investigation of extremely small $x$ region $\left(x<10^{-5}\right)$ at sufficiently large $Q^{2}\left(>10 \mathrm{GeV}^{2}\right)$, where saturation of parton density should manifest itself, is crucial for understanding of QCD basics. Smallest achievable $x$ at $l p$ colliders is given by $Q^{2} / S$. For LHeC with $\sqrt{s}=1.3 \mathrm{TeV}$ minimal achievable value is $x=$ $6 \times 10^{-6}$. In Table 9, we present smallest $x$ values for different SppC based lepton-proton colliders $\left(E_{p}\right.$ is chosen as $\left.68 \mathrm{TeV}\right)$. It is seen that proposed machines has great potential for enlightening of QCD basics.

3.2. Color Octet Leptons. Color octet leptons $\left(l_{8}\right)$ are predicted in preonic models with colored preons [31]. There are various phenomenological studies on $l_{8}$ at $\mathrm{TeV}$ energy scale colliders [32-39]. Resonant production of color octet electron $\left(e_{8}\right)$ and muon $\left(\mu_{8}\right)$ at the FCC based $l p$ colliders (http://collider-reach.web.cern.ch/collider-reach) have been considered in [40] and [41], respectively. Performing similar analyses for SppC based $l p$ colliders we obtain mass discovery limits for $e_{8}$ and $\mu_{8}$ in $\Lambda=M_{l_{8}}$ case (where $\Lambda$ is compositeness scale) which are presented in Figures 2 and 3, respectively. Discovery mass limit value for LHC and SppC is obtained by rescaling ATLAS/CMS second-generation LQ results [42, 43] using the method developed by Salam and Weiler [44]. For lepton colliders, it is obvious that discovery mass limit for pair production of $l_{8}$ is approximately half of CM energies. It is seen that $l_{8}$ search potential of SppC based $l p$ colliders overwhelmingly exceeds that of LHC and lepton colliders. Moreover $l p$ colliders will give an opportunity to determine compositeness scale (for details see $[40,41]$ ).

It should be noted that FCC/SppC based $l p$ colliders have great potential for search of a lot of BSM phenomena, such as excited leptons (see [45] for $\mu^{*}$ ), contact interactions, and R-parity violating SUSY.

\section{Conclusion}

It is shown that construction of linear $e^{+} e^{-}$colliders (or dedicated linac) and muon colliders (or dedicated muon ring) 


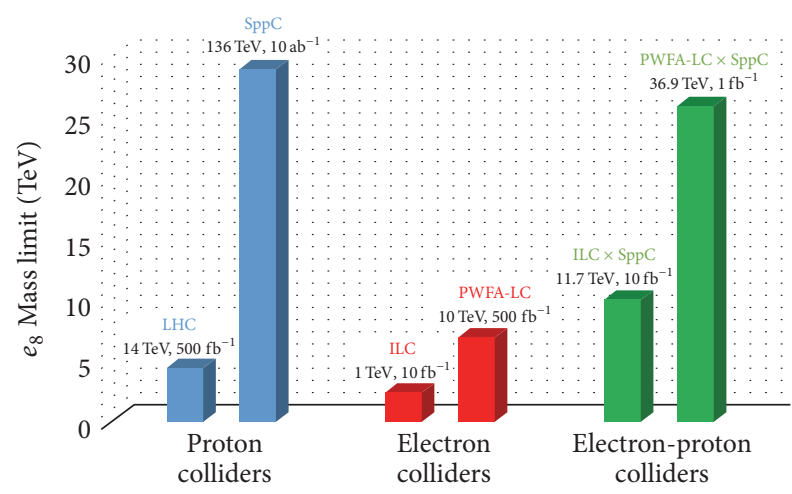

Figure 2: Discovery mass limits for color octet electron at different pp, $e^{+} e^{-}$, and ep colliders.

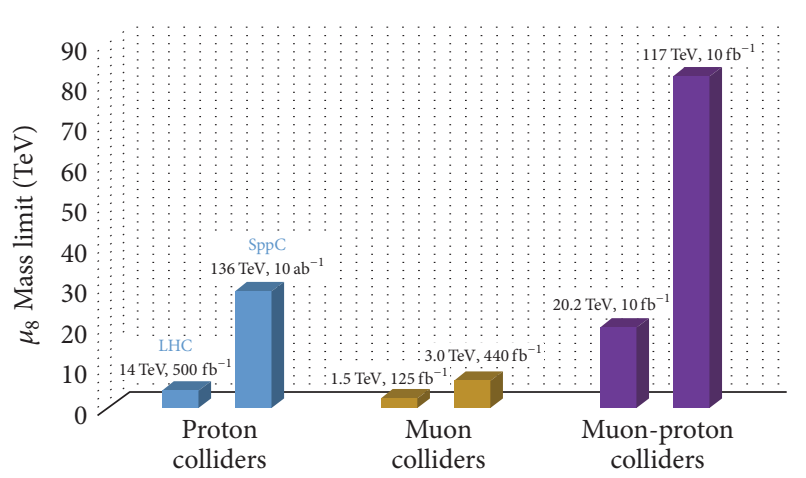

FIGURE 3: Discovery mass limits for color octet muon at different pp, $\mu^{+} \mu^{-}$, and $\mu p$ colliders.

tangential to the SppC will give opportunity to handle leptonproton collisions with multi-TeV CM energies and sufficiently high luminosities. Concerning SM physics, these machines will certainly shed light on QCD basics. BSM search potential of $l p$ colliders essentially exceeds that of corresponding lepton colliders. Also these types of colliders exceed the search potential of the SppC itself for a lot of BSM phenomena.

Acceleration of ion beams at the SppC will give opportunity to provide multi- $\mathrm{TeV}$ center of mass energy in $e A$ and $\mu A$ collisions. In addition, electron beam can be converted to high energy photon beam using Compton backscattering of laser photons which will give opportunity to construct $\mathrm{LC} \otimes \mathrm{SppC}$ based $\gamma p$ and $\gamma A$ colliders. Studies on these topics are ongoing.

In conclusion, systematic study of accelerator, detector, and physics search potential issues of the SppC based ep, $e A$, $\gamma p, \gamma A, \mu p$, and $\mu A$ colliders are essential to foresee the future of particle physics. Certainly, realization of these machines depends on the future results from the LHC as well as FCC and/or SppC.

\section{Conflicts of Interest}

The authors declare that they have no conflicts of interest.

\section{Acknowledgments}

This study is supported by TUBITAK under Grant no. 114F337.

\section{References}

[1] R. Hofstadter and R. W. McAllister, "Electron scattering from the proton," Physical Review, vol. 98, no. 1, pp. 217-218, 1955.

[2] J. I. Friedman and H. W. Kendall, "Deep Inelastic Electron Scattering," Annual Review of Nuclear Science, vol. 22, no. 1, pp. 203-254, 1972.

[3] H. Abramowicz and A. C. Caldwell, "HERA collider physics," Reviews of Modern Physics, vol. 71, no. 5, pp. 1275-1409, 1999.

[4] M. Klein and R. Yoshida, "Collider physics at HERA," Progress in Particle and Nuclear Physics, vol. 61, no. 2, pp. 343-393, 2008.

[5] J. L. Abelleira Fernandez, C. Adolphsen, A. N. Akay et al., "A large hadron electron collider at cern report on the physics and design concepts for machine and detector," Journal of Physics G: Nuclear and Particle Physics, vol. 39, no. 7, Article ID 075001, 2012.

[6] S. Sultansoy, "Linac-ring type colliders: Second way to $\mathrm{TeV}$ scale," The European Physical Journal C-Particles and Fields, vol. 33, pp. 1064-1066, 2004.

[7] A. N. Akay, H. Karadeniz, and S. Sultansoy, "Review of linacring-type collider proposals," International Journal of Modern Physics A, vol. 25, no. 24, pp. 4589-4602, 2010.

[8] Z. Z. Aydin, A. K. Çiftçi, and S. Sultansoy, "Linac-ring type ep machines and $\gamma \mathrm{p}$ colliders based on them," Nuclear Inst. and Methods in Physics Research, A, vol. 351, no. 2-3, pp. 261-265, 1994.

[9] A. Ciftci, S. Sultansoy, Ş. Turkoz, and O. Yavas, "Main parameters of TeV energy $\gamma$ p colliders," Nuclear Instruments and Methods in Physics Research Section A: Accelerators, Spectrometers, Detectors and Associated Equipment, vol. 365, no. 2, pp. 317-328, 1995.

[10] H. Aktaş, N. Büget, A. K. Çiftçi, N. Meriç, S. Sultansoy, and Ö. Yavaş, "New tool for 'old' nuclear physics: FEL $\gamma$-nucleus colliders," Nuclear Instruments and Methods in Physics Research, Section A: Accelerators, Spectrometers, Detectors and Associated Equipment, vol. 428, no. 2, pp. 271-275, 1999.

[11] A. K. Çiftçi, S. Sultansoy, and Ö. Yavaş, "TESLA * HERA based $\gamma \mathrm{p}$ and $\gamma \mathrm{A}$ colliders," Nuclear Instruments and Methods in Physics Research, Section A: Accelerators, Spectrometers, Detectors and Associated Equipment, vol. 472, no. 1-2, pp. 72-78, 2001.

[12] O. Yavas, R. Corsini, H. Braun et al., "CLIC-LHC-based FEL-nucleus collider: Feasibility and physics search potential," Nuclear Instruments and Methods in Physics Research, Section A: Accelerators, Spectrometers, Detectors and Associated Equipment, vol. 552, no. 3, pp. 440-448, 2005.

[13] H. Aksakal, A. K. Ciftci, Z. Nergiz, D. Schulte, and F. Zimmermann, "Conversion efficiency and luminosity for gammaproton colliders based on the LHC-CLIC or LHC-ILC QCD explorer scheme," Nuclear Instruments and Methods in Physics Research, Section A: Accelerators, Spectrometers, Detectors and Associated Equipment, vol. 576, no. 2-3, pp. 287-293, 2007.

[14] E. Arikan and H. Aksakal, "Positron source investigation by using CLIC drive beam for Linac-LHC based $e^{+} p$ collider," Nuclear Instruments and Methods in Physics Research, Section A: Accelerators, Spectrometers, Detectors and Associated Equipment, vol. 683, pp. 63-70, 2012. 
[15] FCC, https://fcc.web.cern.ch.

[16] F. Su, J. Gao, M. Xiao et al., "Method study of parameter choice for a circular proton-proton collider," Chinese Physics C, vol. 40, no. 1, Article ID 017001, 2016.

[17] F. Su, S. Bai, T. Bian et al., "SPPC Parameter Choice and Lattice Design," in Proceedings of the 7th International Particle Accelerator Conference (IPAC'16), pp. 1400-1402, Busan, Korea, May 2016.

[18] Y. C. Acar, A. N. Akay, and S. Beser, "FCC Based Lepton-Hadron and Photon-Hadron Colliders: Luminosity and Physics," https://arxiv.org/abs/1608.02190.

[19] Y. Zhang and Y. Peng, "A High Energy e-p/A Collider Based on CepC-SppC," in Proceedings of the 6th International Particle Accelerator Conference (IPAC'15), Richmond, VA, USA, May 2015.

[20] C. Adolphsen, M. Barone, B. Barish et al., "The International Linear Collider Technical Design Report," https://arxiv.org/abs/ 1306.6328.

[21] J. P. Delahaye, E. Adli, S. J. Gessner et al., "A beam driven plasma-wakefield linear collider from Higgs factory to multiTeV," in Proceedings of the 5th International Particle Accelerator Conference (IPAC '14), pp. 3791-3793, Dresden, Germany, June 2014.

[22] ALOHEP., http://alohep.hepforge.org.

[23] U. Katz, M. Klein, A. Levy, and S. Schlenstedt, The THERA Book, DESY-LC-REV-2001-062, 2001.

[24] R. Brinkmann and M. Dohlus, "A method to overcome the bunch length limitations on $\beta \mathrm{p}^{*}$ for electron-proton colliders," Tech. Rep. DESY-M-95-11, 1995.

[25] I. F. Ginzburg, "Physics at future ep, $\gamma \mathrm{p}$ (linac-ring) and $\mu \mathrm{p}$ colliders," Turkish Journal of Physics, vol. 22, no. 7, pp. 607-610, 1998.

[26] V. D. Shiltsev, "An asymmetric muon-proton collider: luminosity consideration," in Proceedings of the Particle Accelerator Conference, pp. 420-421, Vancouver, Canada, 1997.

[27] S. Sultansoy, "The post-HERA era: brief review of future leptonhadron and photon-hadron colliders," https://arxiv.org/abs/ hep-ph/9911417.

[28] J. P. Delahaye, C. Ankenbrandt, A. Bogacz et al., "Enabling intensity and energy frontier science with a muon accelerator facility in the U.S," https://arxiv.org/abs/1308.0494.

[29] S. Verdú-Andrés, S. Belomestnykh, I. Ben-Zvi, R. Calaga, Q. Wu, and B. Xiao, "Crab cavities for colliders: Past, present and future," Nuclear and Particle Physics Proceedings, vol. 273-275, pp. 193-197, 2016.

[30] B. J. King, "Parameter sets for $10 \mathrm{TeV}$ and $100 \mathrm{TeV}$ muon colliders, and their study at the HEMC'99 workshop," in Proceedings of the AIP Conference Proceedings 530, vol. 122, Montauk, NY, USA, 2000.

[31] I. A. DSouza and C. S. Kalman, PREONS: Models of Leptons, Quarks and Gauge Bosons as Composite Objects, World Scientific Publishing Co. Pte. Ltd., Singapore, Singapore, 1992.

[32] A. Celikel, M. Kantar, and S. Sultansoy, "A search for sextet quarks and leptogluons at the LHC," Physics Letters, Section B: Nuclear, Elementary Particle and High-Energy Physics, vol. 443, no. 1, pp. 359-364, 1998.

[33] T. Mandal and S. Mitra, "Probing color octet electrons at the LHC," Physical Review D - Particles, Fields, Gravitation and Cosmology, vol. 87, no. 9, Article ID 095008, 2013.

[34] D. Gonçalves-Netto, D. López-Val, K. Mawatari, I. Wigmore, and T. Plehn, "Looking for leptogluons," Physical Review
D - Particles, Fields, Gravitation and Cosmology, vol. 87, no. 9, Article ID 094023, 2013.

[35] T. Jelinski and D. Zhuridovy, "Leptogluons in dilepton production at the LHC," Acta Physica Polonica B, vol. 46, no. 11, pp. 2185-2192, 2015.

[36] T. Mandal, S. Mitra, and S. Seth, "Probing compositeness with the CMS eejj\&ejj data," Physics Letters B, vol. 758, pp. 219-225, 2016.

[37] M. Sahin, S. Sultansoy, and S. Turkoz, "Resonant production of color octet electron at the LHeC," Physics Letters, Section B: Nuclear, Elementary Particle and High-Energy Physics, vol. 689, no. 4-5, pp. 172-176, 2010.

[38] M. Sahin, "Resonant production of Spin-3/2 color octet electron at the LHeC," Acta Physica Polonica B, vol. 45, no. 9, pp. 18111831, 2014.

[39] A. N. Akay, H. Karadeniz, M. Sahin, and S. Sultansoy, "Indirect search for color octet electron at next-generation linear colliders," EPL, vol. 95, no. 3, Article ID 31001, 2011.

[40] Y. C. Acar, U. Kaya, B. B. Oner, and S. Sultansoy, "Color octet electron search potential of FCC based e-p colliders," Journal of Physics G: Nuclear and Particle Physics, vol. 44, no. 4, Article ID 045005, p. 045005, 2017.

[41] Y. C. Acar, U. Kaya, and B. B. Oner, "Resonant Production of Color Octet Muons at the Future Circular Collider Based Muon-Proton Colliders," https://arxiv.org/abs/1703.04030.

[42] The ATLAS Collaboration, "Search for scalar leptoquarks in pp collisions at $\sqrt{ } \mathrm{S}=13 \mathrm{TeV}$ with the ATLAS experiment," New Journal of Physics, vol. 18, Article ID 093016, 2016.

[43] The CMS Collaboration, "Search for pair production of first and second generation leptoquarks in proton-proton collisions at $\sqrt{ } \mathrm{S}=8 \mathrm{TeV}$," Physical Review D, vol. 93, no. 3, Article ID 032004, 2016.

[44] G. Salam and A. Weiler, "The collider reach project," http:// collider-reach.web.cern.ch/collider-reach.

[45] A. Caliskan, S. O. Kara, and A. Ozansoy, "Excited muon searches at the FCC-based muon-hadron colliders," Advances in High Energy Physics, vol. 2017, Article ID 1540243, 9 pages, 2017. 

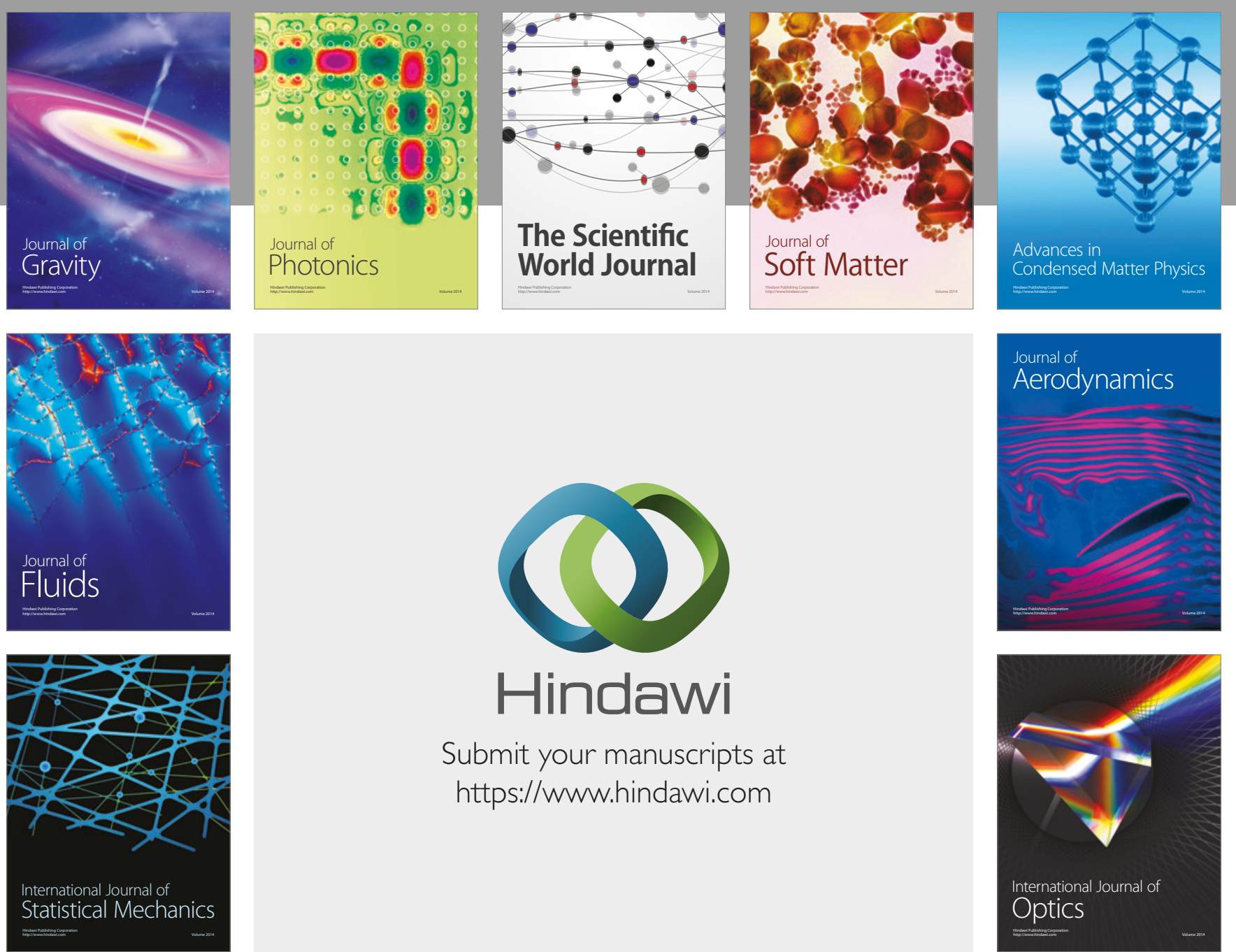

Submit your manuscripts at

https://www.hindawi.com
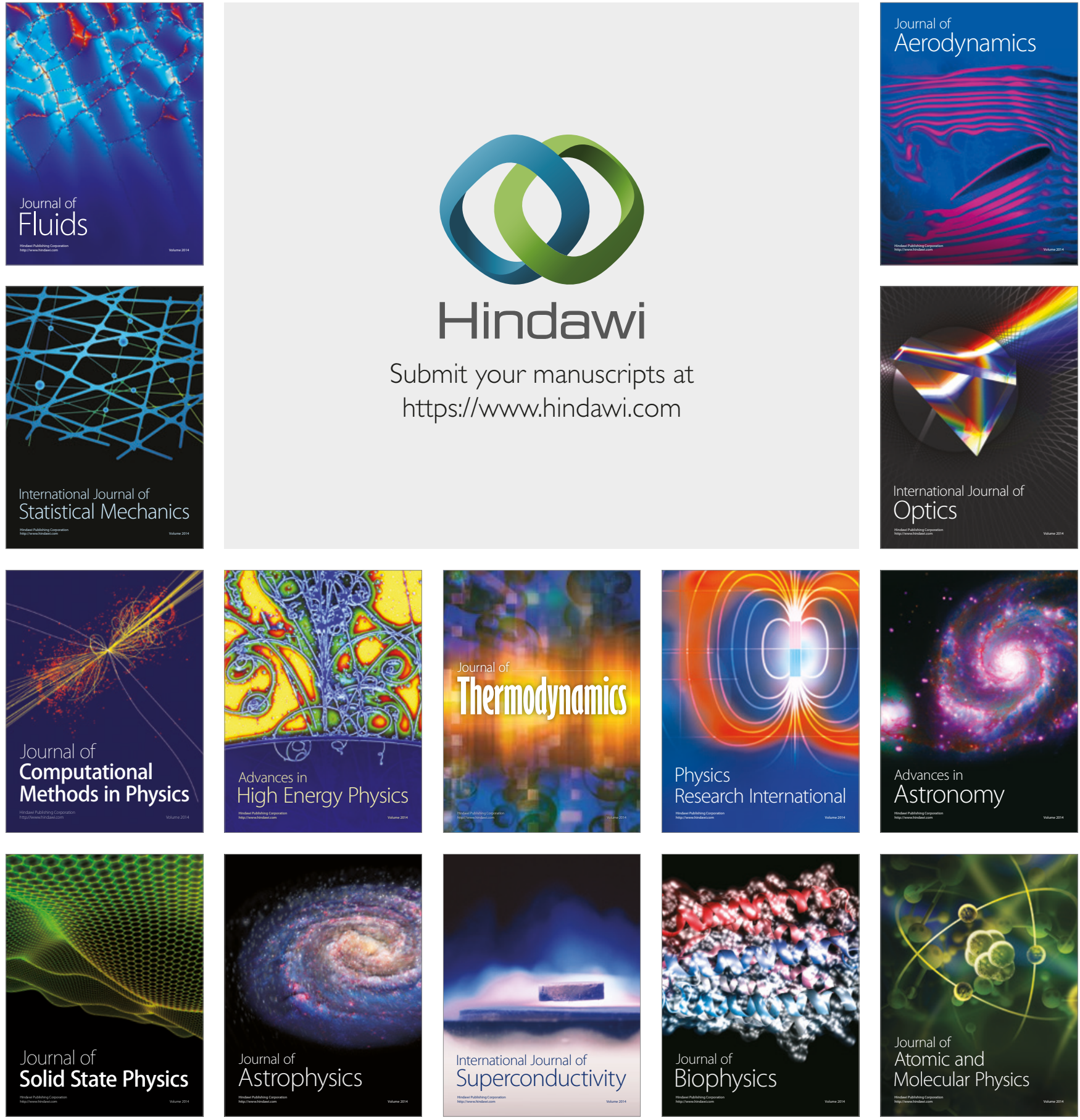